\title{
To Theory One Class Linear Model Noclassical Volterra Type Integral Equation with Left Boundary Singular Point
}

\author{
Nusrat Rajabov \\ Tajik National University, Dushanbe, Tajikistan \\ Email: nusrat38@mail.ru
}

Received April 22, 2013; revised May 22, 2013; accepted June 1, 2013

Copyright (C) 2013 Nusrat Rajabov. This is an open access article distributed under the Creative Commons Attribution License, which permits unrestricted use, distribution, and reproduction in any medium, provided the original work is properly cited.

\begin{abstract}
In this work, we investigate one class of Volterra type integral equation, in model case, when kernels have first order fixed singularity and logarithmic singularity. In detail study the case, when $n=3$. In depend of the signs parameters solution to this integral equation can contain three arbitrary constants, two arbitrary constants, one constant and may have unique solution. In the case when general solution of integral equation contains arbitrary constants, we stand and investigate different boundary value problems, when conditions are given in singular point. Besides for considered integral equation, the solution found cane represented in generalized power series. Some results obtained in the general model case.
\end{abstract}

Keywords: Neoclassical Volterra Type Integral Equation; Left Boundary Singular Point; Boundary Value Problems

\section{Introduction}

Let $\Gamma=\{x: a<x<b\}$ be a set of point on the real axis and consider an integral equation

$$
\varphi(x)+\int_{a}^{x}\left[\sum_{k=1}^{n} p_{k} \ln ^{k-1}\left(\frac{x-a}{t-a}\right)\right] \frac{\varphi(t)}{t-a} \mathrm{~d} t=f(x),
$$

where $p_{j}(1 \leq j \leq n)$ is given constants, $f(x)$ is given function in $\bar{\Gamma}$ and $\varphi(x)$ to be found.

In what follows we in detail go into case $n=3$. In this case the Equation (1) accepts the following form

$$
\begin{aligned}
& \varphi(x)+\int_{a}^{x}\left[p_{1}+p_{2} \ln \left(\frac{x-a}{t-a}\right)+p_{3} \ln ^{2}\left(\frac{x-a}{t-a}\right)\right] \frac{\varphi(t)}{t-a} \mathrm{~d} t \\
& =f(x) .
\end{aligned}
$$

Integral Equation (1) at $p_{2}=0, p_{3}=0$ is model second kind Volterra type singular integral equation with left boundary singular point, theory construction in [1-5]. In the case, when in (1) $p_{3}=0$ Equation (1) investigates in [6].

As $[4,5]$ the solution to this equation is sought in the class of function $\varphi(x) \in C[a, b], \varphi(a)=0$ with following asymptotic behavior

$$
\varphi(x)=o\left[(x-a)^{\mathcal{E}}\right], \mathcal{E}>0 \text { at } x \rightarrow a .
$$

In this case the integrals in the Equation (1) are improper one. Moreover $\varphi(a)=f(a)=0$ i.e. right-hand side is necessarily zero at $x=a$.

In this case in Equation (1) $p_{2}=p_{3}=0$ it investigates in [1]. In this case, in depend from signs $p_{1}$ $\left(p_{1}>0, p_{1}<0\right)$, solution integral Equation (1) is found in explicit form. In this case at $p_{1}<0$ homogeneous integral Equation (1) has one solution and general solution no homogeneous (1) contains one arbitrary constant and at $p_{1}>0$, integral Equation (1) has unique solution.

In case of, when in (1) $p_{3}=0, p_{1} \neq 0, p_{2} \neq 0$ integral Equation (1) investigates in [6]. In this case in depend from corresponding characteristic equation obtained solution integral Equation (1) by two arbitrary constants, one arbitrary constant. Select the case, when integral Equation (1) has unique solution. To problems investigation one dimensional and many-dimensional Volterra type integral equation with fixed boundary and interior singular points and singular domains in kernels dedicate [1-7].

Support that solution integral Equation (1) function $\varphi(x) \in C^{(3)}(\Gamma)$. Besides, let in Equation (1) function $f(x) \in C^{(3)}(\Gamma)$ two. Then differentiating integral Equation (1) three times, we obtained the following third order degeneration differential equation 


$$
\begin{aligned}
& D_{x}^{3} \varphi(x)+p_{1} D_{x}^{2} \varphi(x)+p_{2} D_{x} \varphi(x) p_{3} \varphi(x) \\
& =D_{x}^{3} f(x),
\end{aligned}
$$

where $D_{x}=(x-a) \frac{\mathrm{d}}{\mathrm{d} x}$.

Homogeneous differential Equation (3) is corresponding to the following characteristic equation

$$
\lambda^{3}+p_{1} \lambda^{2} p_{2} \lambda+2 p_{3}=0 .
$$

\section{Representation the General Solution}

\subsection{The Case, When the Roots of the Characteristic Equation Real and Different}

Let in differential Equation (3) parameters $p_{j}(1 \leq j \leq 3)$ suchthat, the roots of the characteristic Equation (4) real and different. Its denote by $\lambda_{1}, \lambda_{2}, \lambda_{3}$. In this case, immediately testing we see that, general solution homogeneous differential Equation (2) is given by formula

$$
\varphi(x)=(x-a)^{\lambda_{1}} C_{1}+(x-a)^{\lambda_{2}} C_{2}+(x-a)^{\lambda_{3}} C_{3},
$$

where $C_{j}(1 \leq j \leq 3)$ arbitrary constants.

When, $\lambda_{j}>0(1 \leq j \leq 3)$, function $\varphi(x)$ definable by formula (5) satisfy homogeneous integral Equation (1). So, function $\varphi(x)$ determined by formula (5) is given general solution homogeneous integral Equation (1).

For obtained the solution non homogeneous integral Equation (1), first time use the variation arbitrary constants methods, we use the general solution of the differential Equation (3). After transformation, we see that, if solution integral Equation (1) in this case exist, then we its my be represented in the following form

$$
\begin{aligned}
\varphi(x) & =(x-a)^{\lambda_{1}} C_{1}+(x-a)^{\lambda_{2}} C_{2}+(x-a)^{\lambda_{3}} C_{3}+f(x)+\frac{1}{\Delta_{0}} \int_{a}^{x}\left[\lambda_{1}^{3}\left(\frac{x-a}{t-a}\right)^{\lambda_{1}}+\lambda_{2}^{3}\left(\frac{x-a}{t-a}\right)^{\lambda_{2}}+\lambda_{3}^{3}\left(\frac{x-a}{t-a}\right)^{\lambda_{3}}\right] \frac{f(t)}{t-a} \mathrm{~d} t \\
& \equiv K_{1}^{-}\left[C_{1}, C_{2}, C_{3}, f(x)\right]
\end{aligned}
$$

where $C_{j}(1 \leq j \leq 3)$ arbitrary constants,

$$
\Delta_{0}=\left|\begin{array}{lll}
1, & 1, & 1 \\
\lambda_{1}, & \lambda_{2}, & \lambda_{3} \\
\lambda_{1}^{2}, & \lambda_{2}^{2}, & \lambda_{3}^{2}
\end{array}\right|=\lambda_{2} \lambda_{3}\left(\lambda_{3}-\lambda_{2}\right)+\lambda_{1} \lambda_{2}\left(\lambda_{2}-\lambda_{1}\right)+\lambda_{1} \lambda_{3}\left(\lambda_{1}-\lambda_{3}\right) .
$$

The solution of the type (6) obtained in the case, when $f(x) \in C^{(3)}(\Gamma), f(a)=0$, solution integral equation (1), function $\varphi(x)$ exist and belong to Class $C^{(3)}(\Gamma)$.

Immediately testing, we see that, of $\lambda_{j}>0(1 \leq j \leq 3)$, $f(x) \in C^{(3)}(\Gamma), f(a)=0$ with asymptotic behavior

$$
\begin{aligned}
& f(x)=o\left[(x-a)^{\delta_{1}}\right], \delta_{1}>\lambda, \\
& \lambda=\max \left(\lambda_{1}, \lambda_{2}, \lambda_{3}\right) \text { at } x \rightarrow a
\end{aligned}
$$

then function (5) satisfied Equation (1).

Be valid the following confirmation.

Theorem 1. Let in integral Equation (1) parameters $p_{j}(1 \leq j \leq 3)$ such that, the roots of the algebraic Equation (4) real, different and positive, function $f(x) \in$ $C(\bar{\Gamma}), f(a)=0$ with asymptotic behavior (7). Then integral Equation (1) in class of function $\varphi(x) \in C(\bar{\Gamma})$ vanishing in point $x=a$ is always solvability and its solution is given by formula $(6), C_{j}(1 \leq j \leq 3)$ are arbitrary constants.

Characteristics 1. Let in integral Equation (1) parameters $p_{j}(1 \leq j \leq 3)$, function $f(x)$ satisfy any condition of theorem 1.Then, from (6) it follows, the solution integral Equation (1) $\varphi(x) \in C(\bar{\Gamma}), \varphi(a)=0$ with following asymptotic behavior

$$
\varphi(x)=o\left[(x-a)^{\mu_{1}}\right], \mu_{1}=\min \left(\lambda_{1}, \lambda_{2}, \lambda_{3}\right), \text { at } x \rightarrow a .
$$

If, the roots of the characteristic Equation (4) real, different and $\lambda_{1}<0, \lambda_{2}>0, \lambda_{3}>0$, then it follows, from formula (6) $C_{1}=0$.

In this case, if exist the solution integral Equation (1), then it is possible is represent in following form

$$
\begin{aligned}
\varphi(x) & =(x-a)^{\lambda_{2}} C_{4}+(x-a)^{\lambda_{3}} C_{5}+f(x)+\frac{1}{\Delta_{0}} \int_{a}^{x}\left[-\left|\lambda_{1}\right|^{3}\left(\frac{t-a}{x-a}\right)^{\left|\lambda_{1}\right|}+\lambda_{2}^{3}\left(\frac{x-a}{t-a}\right)^{\lambda_{2}}+\lambda_{3}^{3}\left(\frac{x-a}{t-a}\right)^{\lambda_{3}}\right] \frac{f(t)}{t-a} \mathrm{~d} t \\
& \equiv K_{2}^{-}\left[C_{4}, C_{5}, f(x)\right]
\end{aligned}
$$

where $C_{4}, C_{5}$-are arbitrary constants.

The solution of the type (8) exist, if $f(x) \in C(\bar{\Gamma})$, $f(a)=0$ with asymptotic behavior

$$
\begin{aligned}
& f(x)=o\left[(x-a)^{\delta_{2}}\right], \delta_{2}>\mu_{1}, \\
& \mu_{1}=\min \left(\lambda_{2}, \lambda_{3}\right) \text { at } x \rightarrow a
\end{aligned}
$$


So, in this case have the following confirmation.

Theorem 2. Let in integral Equation (1) parameters $p_{j}(1 \leq j \leq 3)$ such that, the roots of the algebraic Equation (4) real, different and also $\lambda_{1}<0, \lambda_{2}>0$, $\lambda_{3}>0, f(x) \in C(\bar{\Gamma}), f(a)=0$ with asymptotic behavior (9). Then integral Equation (1) in class of function $\varphi(x) \in C(\bar{\Gamma})$ vanishing in point $x=a$ is always solvability and its solution is given by formula (8), $C_{j}(j=4,5)$ are arbitrary constants.

Characteristics 2. Let in integral Equation (1) parameters $p_{j}(1 \leq j \leq 3)$, function $f(x)$ satisfy any condition of theorem 2 . Then, from (8) it follows, the solution integral Equation (1) $\varphi(x) \in C(\bar{\Gamma}), \varphi(a)=0$ with following asymptotic behavior

$$
\varphi(x)=o\left[(x-a)^{\mu_{2}}\right], \mu_{2}=\min \left(\lambda_{2}, \lambda_{3}\right), \text { at } x \rightarrow a
$$

Remark 1. Confirmation similar to theorem 2 obtained and in the following cases:

a) $\lambda_{1}>0, \lambda_{2}<0, \lambda_{3}>0$; b) $\lambda_{1}>0, \lambda_{2}>0, \lambda_{3}<0$.

If the roots of the characteristic equation (4) real and different, $\lambda_{1}<0, \lambda_{2}<0, \lambda_{3}>0$, then from integral representation (6), follows, that in order that $\varphi(x)$ is solution integral Equation (1) in this case, it is necessary $C_{1}=C_{2}=0$. In this case, if exist solution integral Equation (1), then it will be represented in following form

$$
\begin{aligned}
\varphi(x) & =(x-a)^{\lambda_{3}} C_{6}+f(x)+\frac{1}{\Delta_{0}} \int_{a}^{x}\left[\lambda^{3}\left(\frac{t-a}{x-a}\right)^{\left|\lambda_{1}\right|}+\lambda_{2}^{3}\left(\frac{t-a}{x-a}\right)^{\left|\lambda_{2}\right|}+\lambda_{3}^{3}\left(\frac{x-a}{t-a}\right)^{\lambda_{3}}\right] \frac{f(t)}{t-a} \mathrm{~d} t \\
& \equiv K_{2}^{-}\left[C_{6}, f(x)\right]
\end{aligned}
$$

where $C_{6}$ are arbitrary constant.

The solution of the type (10) exist, if $f(x) \in C(\bar{\Gamma})$, $f(a)=0$ with asymptotic behavior

$$
f(x)=o\left[(x-a)^{\delta_{3}}\right], \delta_{3}>\lambda_{3} \text { at } x \rightarrow a .
$$

So, we proof.

The following confirmation.

Theorem 3. Let in integral Equation (1) parameters $p_{j}(1 \leq j \leq 3)$ such that, the roots of the algebraic Equation (4) real, different and also $\lambda_{1}<0, \lambda_{2}<0$, $\lambda_{3}>0, f(x) \in C(\bar{\Gamma}), f(a)=0$ with asymptotic behavior (11). Then integral Equation (1) in class of function $\varphi(x) \in C(\bar{\Gamma})$ vanishing in point $x=a$ is always solvability and its solution is given by formula (10), where $C_{3}$ are arbitrary constant.

Characteristics 3. Let in integral Equation (1) pa- rameters $p_{j}(1 \leq j \leq 3)$, function $f(x)$ satisfy any condition of theorem 3 . Then the solution of the integral Equation (1) in point $x=a$ vanish and its asymptotic behavior determined from formula

$$
\varphi(x)=o\left[(x-a)^{\lambda_{3}}\right] \text { at } x \rightarrow a .
$$

Remark 2. Confirmation similar to theorem 3, obtained and in the following cases:

a) $\lambda_{1}>0, \lambda_{2}<0, \lambda_{3}<0$; b) $\lambda_{1}<0, \lambda_{2}>0, \lambda_{3}<0$.

If the roots of the characteristic Equation (4) real, different and $\lambda_{j}<0(1 \leq j \leq 3)$, then from integral representation (6) follows, in order that $\varphi(x)$ is solution integral Equation (1) in this case, it is necessary $C_{1}=C_{2}$ $=C_{3}=0$. In this case, if exist solution integral Equation (1), then its will be represented in form

$$
\varphi(x)=f(x)+\frac{1}{\Delta_{0}} \int_{a}^{x}\left[\lambda_{1}^{3}\left(\frac{t-a}{x-a}\right)^{\left|\lambda_{1}\right|}+\lambda_{2}^{3}\left(\frac{t-a}{x-a}\right)^{\left|\lambda_{2}\right|}+\lambda_{3}^{3}\left(\frac{t-a}{x-a}\right)^{\left|\lambda_{3}\right|}\right] \frac{f(t)}{t-a} \mathrm{~d} t \equiv K_{4}^{-}[f(x)]
$$

The solution of the type (12) exist, if $f(x) \in C(\bar{\Gamma})$, $f(a)=0$ with asymptotic behavior

$$
f(x)=o\left[(x-a)^{\varepsilon}\right], \varepsilon>0 \text { at } x \rightarrow a
$$

So we proof the following confirmation.

Theorem 4. Let in integral Equation (1) parameters $p_{j}(1 \leq j \leq 3)$ such that, the roots of the algebraic Equation (4) real, different and positive. The function $f(x) \in C(\bar{\Gamma}), f(a)=0$ with asymptotic behavior (13). Then integral Equation (1) in class of function $\varphi(x) \in C(\bar{\Gamma})$ vanishing in point $x=a$ have unique solution, which give by formula (12).

Characteristics 4. Let in integral Equation (1) pa- rameters $p_{j}(1 \leq j \leq 3)$, function $f(x)$ satisfy any condition of theorem 4 . Then the solution of the integral Equation (1) in point $x=a$ vanish and its asymptotic behavior determined from formula

$$
\varphi(x)=o\left[(x-a)^{\varepsilon}\right], \varepsilon>0 \text { at } x \rightarrow a .
$$

\subsection{The Case, When the Roots of the Characteristic Equation Real and Equal}

Let in integral Equation (1) parameters $p_{j}(1 \leq j \leq 3)$, such that, the roots of the characteristic Equation (4) real and equal.

In this case we have the following confirmation: 
Theorem 5. Let in integral Equation (1) parameters $p_{j}(1 \leq j \leq 3)$ such that, the roots of the characteristic Equation (4) real, equal and positive, that is $\lambda_{1}=\lambda_{2}=$ $\lambda_{3}=\lambda>0$. Assume that a function $f(x) \in C(\bar{\Gamma})$, $f(a)=0$ with the following asymptotic behavior

$$
f(x)=o\left[(x-a)^{\delta_{4}}\right], \delta_{4}>\lambda \text {, at } x \rightarrow a .
$$

Then homogeneous integral Equation (1) in class of function $\varphi(x) \in C(\bar{\Gamma})$ vanishing in point $x=a$, have three linear independent solutions the type

$$
\begin{gathered}
\varphi_{1}(x)=(x-a)^{\lambda}, \quad \varphi_{2}(x)=(x-a)^{\lambda} \ln (x-a), \\
\varphi_{3}(x)=(x-a)^{\lambda} \ln ^{2}(x-a) .
\end{gathered}
$$

Non homogeneous integral Equation (1), always solvable. Its general solution contain three arbitrary constant and given by formula

$$
\begin{aligned}
\varphi(x)= & (x-a)^{\lambda}\left[C_{1}+\ln (x-a) C_{2}+\ln ^{2}(x-a) C_{3}\right]+f(x) \\
& +\frac{\lambda}{2} \int_{a}^{x}\left[6+6 \lambda \ln \left(\frac{x-a}{t-a}\right)+\lambda^{2} \ln ^{2}\left(\frac{x-a}{t-a}\right)\right]\left(\frac{x-a}{t-a}\right)^{\lambda} \frac{f(t)}{t-a} \mathrm{~d} t \\
\equiv & K_{5}^{-}\left[C_{1}, C_{2}, C_{3}, f(x)\right],
\end{aligned}
$$

Were $C_{j}(1 \leq j \leq 3)$-arbitrary constants.

Characteristics 5. In this case, when in integral Equation (1) parameters $p_{j}(1 \leq j \leq 3)$, function $f(x)$ satisfy any condition of theorem 5 , then solution integral Equation (1) in point $x=a$ vanish and its asymptotic behavior determined from formula

$$
\begin{gathered}
\varphi(x)=o\left[(x-a)^{\lambda-\varepsilon}\right], \\
\varepsilon>0, \text { at } x \rightarrow a .
\end{gathered}
$$

From integral representation (14) follows. If solution integral Equation (1) at $\lambda_{1}=\lambda_{2}=\lambda_{3}=\lambda<0$ exist, then we may be represented it's in form

$$
\varphi(x)=f(x)+\frac{\lambda}{2} \int_{a}^{x}\left[6+6 \lambda \ln \left(\frac{x-a}{t-a}\right)+\lambda^{2} \ln ^{2}\left(\frac{x-a}{t-a}\right)\right]\left(\frac{t-a}{x-a}\right)^{|\lambda|} \frac{f(t)}{t-a} \mathrm{~d} t \equiv K_{6}^{-}[f(x)] .
$$

The solution of the type (15) exist, if $f(x) \in C(\bar{\Gamma})$, $f(a)=0$ with the following asymptotic behavior

$$
f(x)=o\left[(x-a)^{\varepsilon}\right], \varepsilon>0, \text { at } x \rightarrow a .
$$

So in the case, when $\lambda_{1}=\lambda_{2}=\lambda_{3}=\lambda<0$, proof the following confirmation.

Theorem 6. Let in integral Equation (1) parameters $p_{j}(1 \leq j \leq 3)$ such that, the all roots of the characteristic Equation (4) real, equal and negative, that is $\lambda_{1}=\lambda_{2}$ $=\lambda_{3}=\lambda<0$. Assume that a function $f(x) \in C(\bar{\Gamma})$, $f(a)=0$ with the asymptotic behavior (16). Then, integral Equation (1) in class $C(\bar{\Gamma})$ have unique solution and give by formula (15).

Characteristics 6. In this case, when fulfillment any condition theorem 6 , then solution integral equation in point $x=a$ vanish and its asymptotic behavior determined from formula

$$
\begin{aligned}
\varphi(x)=o\left[(x-a)^{\varepsilon}\right], \varepsilon>0, \text { at } x \rightarrow a . & \text { it will be represented in form } \\
\varphi(x)= & (x-a)^{\lambda_{1}} C_{1}+(x-a)^{A}\left[C_{2} \cos [B \ln (x-a)]+C_{3} \sin [B \ln (x-a)]\right]+f(x) \\
& -\frac{1}{\Delta_{0}} \int_{a}^{x}\left\{\lambda_{1}^{3} B\left(\frac{x-a}{t-a}\right)^{\lambda_{1}}+\left(\frac{x-a}{t-a}\right)^{A} D_{1} \sin \left[B \ln \left(\frac{x-a}{t-a}\right)\right]+D_{2} \cos \left[B \ln \left(\frac{x-a}{t-a}\right)\right]\right\} \frac{f(t)}{t-a} \mathrm{~d} t \\
\equiv & K_{7}^{-}\left[C_{1}, C_{2}, C_{3}, f(x)\right]
\end{aligned}
$$

\subsection{The Case, When One Roots of the} Characteristic Equation Real and Two the Roots of the Characteristic Equation Complex and Conjugate

Let in integral Equation (1) parameters $p_{j}(1 \leq j \leq 3)$ such that, the one roots of characteristic Equation (4) real and two the roots of the characteristic equation complex conjugate. Correspondingly its denote by $\lambda_{1}, \lambda_{2}=A+i B$, $\lambda_{3}=A-i B$. When $\lambda_{1}>0, A>0$, then by this roots corresponding following particular solution homogeneous integral Equation (1):

$$
\begin{aligned}
& \varphi_{1}(x)=(x-a)^{\lambda_{1}}, \\
& \varphi_{2}(x)=(x-a)^{A} \cos [B \ln (x-a)] \\
& \varphi_{3}(x)=(x-a)^{A} \sin [B \ln (x-a)] .
\end{aligned}
$$

In this case, if solution integral Equation (1) exist, then 
where

$$
\begin{gathered}
\Delta_{0}=2 A B \lambda_{1}-\lambda_{1}^{2} B-B\left(A^{2}+B^{2}\right) \neq 0, \\
D_{1}=B^{4}-A^{4}-A \lambda_{1}\left(3 B^{2}-A^{2}\right), \\
D_{2}=2 A B\left(A^{2}+B^{2}\right)+B \lambda_{1}\left(B^{2}-3 A^{2}\right) .
\end{gathered}
$$

The solution of the type (18) exist, if $\lambda_{1}>0, A>0$, $f(x) \in C(\bar{\Gamma}), f(a)=0$ with the following asymptotic behavior

$$
f(x)=o\left[(x-a)^{\delta_{5}}\right], \delta_{5}>\max \left(\lambda_{1}, A\right) \text { at } x \rightarrow a .
$$

So in this case we have the following confirmation.

Theorem 7. Let in integral Equation (1) parameters $p_{j}(1 \leq j \leq 3)$ such that, one the roots of the characteristic Equation (4) real positive, two out of its complex conjugate $\left(\lambda_{2}=A+i B, \lambda_{3}=A-i B\right)$. Besides let $A=\operatorname{Real} \lambda_{2}>0$.
Assume that a function $f(x) \in C(\bar{\Gamma}), f(a)=0$ with asymptotic behavior (19). Then homogeneous integral Equation (1), in class $C(\bar{\Gamma})$ vanishing in point $x=a$, has three linear Independent solution of the type (17). Non homogenous integral Equation (1) always solvable, its general solution contain three arbitrary constants and given by formula (18), where $C_{j}(1 \leq j \leq 3)$-arbitrary constants.

Characteristics 7. In the case, when fulfillment any condition theorem 7, then solution integral Equation (1) in point $x=a$ vanish and its behavior determined from following asymptotic formula

$$
\varphi(x)=o\left[(x-a)^{\delta_{6}}\right], \delta_{6}>\min \left(\lambda_{1}, \mathrm{~A}\right) \text { at } x \rightarrow a .
$$

From integral representation (18) follows, if the roots of the algebraic Equation (4) satisfy condition of the theorem 7, besides $\lambda_{1}>0, A>0$. If $\lambda_{1}<0, A>0$, then if exist solution integral Equation (1) in this case, then its represented in following form

$$
\begin{aligned}
\varphi(x)= & (x-a)^{A}\left[C_{2} \cos [B \ln (x-a)]+C_{3} \sin [B \ln (x-a)]\right]+f(x) \\
& -\frac{1}{\Delta_{0}} \int_{a}^{x} \lambda_{1}^{3}\left\{B\left(\frac{t-a}{x-a}\right)^{\left|\lambda_{1}\right|}+\left(\frac{x-a}{t-a}\right)^{A}\left[D_{1} \sin \left[B \ln \left(\frac{x-a}{t-a}\right)\right]+D_{2} \cos \left[B \ln \left(\frac{x-a}{t-a}\right)\right]\right]\right\} \frac{f(t)}{t-a} \mathrm{~d} t \\
\equiv & K_{8}^{-}\left[C_{2}, C_{3}, f(x)\right] .
\end{aligned}
$$

In this case for convergence integrals in right part (20), necessary $f(x) \in C(\bar{\Gamma}), \quad f(a)=0$ with asymptotic behavior

$$
f(x)=o\left[(x-a)^{\delta_{7}}\right], \delta_{7}>A \text { at } x \rightarrow a .
$$

So, we proof. the following confirmation.

Theorem 8. Let in integral Equation (1) parameters $p_{j}(1 \leq j \leq 3)$ satisfy condition theorem 7 , besides $\lambda_{1}>$ $0, A>0$. Let $\lambda_{1}<0, A>0$. Function $f(x) \in C(\bar{\Gamma})$, $f(a)=0$ with asymptotic behavior (21). Then homogeneous integral Equation (1), in class $C(\bar{\Gamma})$ vanishing in point $x=a$, has two linear Independent solution

$$
\begin{array}{r}
\varphi_{2}(x)=(x-a)^{A} \cos [B \ln (x-a)], \\
\varphi_{3}(x)=(x-a)^{A} \sin [B \ln (x-a)] . \\
=(x-a)^{\lambda_{1}} C_{1}+f(x)-\frac{1}{\Delta_{0}} \int_{a}^{x}\left\{\lambda_{1}^{3} B\left(\frac{x-a}{t-a}\right)^{\lambda_{1}}\right. \\
\equiv K_{9}^{-}\left[C_{1}, f(x)\right] .
\end{array}
$$$$
\varphi(x)=(x-a)^{\lambda_{1}} C_{1}+f(x)-\frac{1}{\Delta_{0}} \int_{a}^{x}\left\{\lambda_{1}^{3} B\left(\frac{x-a}{t-a}\right)^{\lambda_{1}}+\left(\frac{t-a}{x-a}\right)^{|A|}\left[D_{1} \sin \left[B \ln \left(\frac{x-a}{t-a}\right)\right]+D_{2} \cos \left[B \ln \left(\frac{x-a}{t-a}\right)\right]\right]\right\} \frac{f(t)}{t-a} \mathrm{~d} t
$$

Non homogenous integral Equation (1) always solvable and its general solution from class $C(\bar{\Gamma})$ is given by formula (20), where $C_{j}(2 \leq j \leq 3)$-arbitrary constants.

Characteristics 8. In the case, when fulfillment any condition theorem 8, then solution integral Equation (1) in point $x=a$ vanish and its behavior determined from following asymptotic formula

$$
\varphi(x)=o\left[(x-a)^{A}\right] \text { at } x \rightarrow a .
$$

Now suppose, that the roots of the algebraic Equation (4) satisfy condition of the theorem 7, besides $\lambda_{1}>0$, $A>0$. Let $\lambda_{1}>0, A<0$. Then, if exist solution integral Equation (1) in this case, then its represented in following form
In this case for convergence integrals in right part (22), necessary $f(x) \in C(\bar{\Gamma}), \quad f(a)=0$ with asymptotic behavior

$$
f(x)=o\left[(x-a)^{\delta_{8}}\right], \delta_{8}>\lambda_{1} \text { at } x \rightarrow a .
$$

So, we proof. the following confirmation. 
Theorem 9. Let in integral Equation (1) parameters $p_{j}(1 \leq j \leq 3)$ satisfy condition theorem 7 , besides $\lambda_{1}>$ $0, A>0$. Let $\lambda_{1}>0, A<0$. Function $f(x) \in C(\bar{\Gamma})$, $f(a)=0$ with asymptotic behavior (23). Then homogeneous integral Equation (1), in class $C(\bar{\Gamma})$ vanishing in point $x=a$, one solution $\varphi_{1}(x)=(x-a)^{\lambda_{1}}$. Non homogenous integral Equation (1) always solvable and its general solution from class $C(\bar{\Gamma})$ is given by formula (22), where $C_{1}$-arbitrary constant.

Characteristics 9. In the case, when fulfillment any condition theorem 9, then solution integral Equation (1) in point $x=a$ vanish and its behavior determined from following asymptotic formula

$$
\begin{gathered}
\varphi(x)=o\left[(x-a)^{\lambda_{1}}\right], \\
\text { at } x \rightarrow a
\end{gathered}
$$

In the case, when $\lambda_{1}<0, A<0$, then from integral representation (18) follows, that, if exidt solution integral Equation (1) in this case, then it is possible in following form

$$
\begin{aligned}
\varphi(x) & =f(x)-\frac{1}{\Delta_{0}} \int_{a}^{x}\left\{\lambda_{1}^{3} B\left(\frac{t-a}{x-a}\right)^{||_{1} \mid}+\left(\frac{t-a}{x-a}\right)^{|A|}\left[D_{1} \sin B\left[\ln \left(\frac{x-a}{t-a}\right)\right]+D_{2} \cos B\left[\ln \left(\frac{x-a}{t-a}\right)\right]\right]\right\} \frac{f(t)}{t-a} \mathrm{~d} t \\
& \equiv K_{10}^{-}[f(x)] .
\end{aligned}
$$

In this case for convergence integrals in right part (24), it is sufficient $f(x) \in C(\bar{\Gamma}), f(a)=0$ with asymptotic behavior

$$
f(x)=o\left[(x-a)^{\varepsilon}\right], \varepsilon>0, \text { at } x \rightarrow a .
$$

So, we proof. the following confirmation.

Theorem 10. Let in integral Equation (1) parameters $p_{j}(1 \leq j \leq 3)$ satisfy condition theorem 7 , besides $\lambda_{1}>$ $0, A>0$. Let $\lambda_{1}<0, A<0$. Function $f(x) \in C(\bar{\Gamma})$, $f(a)=0$ with asymptotic behavior (25). Then integral Equation (1), in class $C(\bar{\Gamma})$ vanishing in point $x=a$, have unique solution, which given by formula (24).
Characteristics 10. In the case, when fulfillment any condition theorem 10 , then solution integral equation (1) in point $x=a$ vanish and its behavior determined from following asymptotic formula

$$
\varphi(x)=o\left[(x-a)^{\varepsilon}\right], \varepsilon>0 \text { at } x \rightarrow a
$$

\section{Property of the Solution}

Let fulfillment any condition of the theorem 1. Differentiating the solution of the type (6), immediate verification, we can easily convince to correctness of the following equality:

$$
\begin{aligned}
D_{x}^{a} \varphi(x)= & \lambda_{1}(x-a)^{\lambda_{1}} C_{1}+\lambda_{2}(x-a)^{\lambda_{2}} C_{2}+\lambda_{3}(x-a)^{\lambda_{3}} C_{3}+D_{x}^{a} f(x) \\
& +\frac{\lambda_{1}^{3}+\lambda_{2}^{3}+\lambda_{3}^{3}}{\Delta_{0}} f(x)+\frac{1}{\Delta_{0}} \int_{a}^{x}\left[\lambda_{1}^{4}\left(\frac{x-a}{t-a}\right)^{\lambda_{1}}+\lambda_{2}^{4}\left(\frac{x-a}{t-a}\right)^{\lambda_{2}}+\lambda_{3}^{4}\left(\frac{x-a}{t-a}\right)^{\lambda_{3}}\right] \frac{f(t)}{t-a} \mathrm{~d} t,
\end{aligned}
$$

where $D_{x}^{a}=(x-a) \frac{\mathrm{d}}{\mathrm{d} x}$.

In an analogous way differentiating the expression (26), we have

$$
\begin{aligned}
\left(D_{x}^{a}\right)^{2} \varphi(x)= & \lambda_{1}^{2}(x-a)^{\lambda_{1}} C_{1}+\lambda_{2}^{2}(x-a)^{\lambda_{2}} C_{2}+\lambda_{3}^{2}(x-a)^{\lambda_{3}} C_{3}+\left(D_{x}^{a}\right)^{2} f(x)+\frac{\lambda_{1}^{3}+\lambda_{2}^{3}+\lambda_{3}^{3}}{\Delta_{0}} D_{x}^{a} f(x) \\
& +\frac{\lambda_{1}^{4}+\lambda_{2}^{4}+\lambda_{3}^{4}}{\Delta_{0}} f(x) \frac{1}{\Delta_{0}} \int_{a}^{x}\left[\lambda_{1}^{5}\left(\frac{x-a}{t-a}\right)^{\lambda_{1}}+\lambda_{2}^{5}\left(\frac{x-a}{t-a}\right)^{\lambda_{2}}+\lambda_{3}^{5}\left(\frac{x-a}{t-a}\right)^{\lambda_{3}}\right] \frac{f(t)}{t-a} \mathrm{~d} t .
\end{aligned}
$$

From Equality (6), (26), (27) we find

$$
\begin{aligned}
& C_{1}=\frac{\left(\lambda_{3}-\lambda_{2}\right)}{\Delta_{0}} \lim _{x \rightarrow a}\left\{(x-a)^{-\lambda_{1}}\left[\left(D_{x}^{a}\right)^{2} \varphi(x)-\left(\lambda_{2}+\lambda_{3}\right) D_{x}^{a} \varphi(x)+\lambda_{2} \lambda_{3} \varphi(x)\right]\right\} \equiv \frac{\left(\lambda_{3}-\lambda_{2}\right)}{\Delta_{0}} \lim _{x \rightarrow a} T_{x}^{1} \varphi(x), \\
& C_{2}=\frac{\left(\lambda_{1}-\lambda_{3}\right)}{\Delta_{0}} \lim _{x \rightarrow a}\left\{(x-a)^{-\lambda_{2}}\left[\left(D_{x}^{a}\right)^{2} \varphi(x)-\left(\lambda_{1}+\lambda_{3}\right) D_{x}^{a} \varphi(x)+\lambda_{1} \lambda_{3} \varphi(x)\right]\right\} \equiv \frac{\left(\lambda_{1}-\lambda_{3}\right)}{\Delta_{0}} \lim _{x \rightarrow a} T_{x}^{2} \varphi(x), \\
& C_{3}=\frac{\left(\lambda_{2}-\lambda_{1}\right)}{\Delta_{0}} \lim _{x \rightarrow a}\left\{(x-a)^{-\lambda_{3}}\left[\left(D_{x}^{a}\right)^{2} \varphi(x)-\left(\lambda_{1}+\lambda_{2}\right) D_{x}^{a} \varphi(x)+\lambda_{1} \lambda_{2} \varphi(x)\right]\right\} \equiv \frac{\left(\lambda_{2}-\lambda_{1}\right)}{\Delta_{0}} \lim _{x \rightarrow a} T_{x}^{3} \varphi(x) .
\end{aligned}
$$


Differentiating the solution of the type (8), immediate verification, we can easily convince to correctness of the following equality:

$$
\begin{aligned}
D_{x}^{a} \varphi(x)= & \lambda_{2}(x-a)^{\lambda_{2}} C_{4}+\lambda_{3}(x-a)^{\lambda_{3}} C_{5}+D_{x}^{a} f(x)+\frac{\lambda_{1}^{3}+\lambda_{2}^{3}+\lambda_{3}^{3}}{\Delta_{0}} f(x) \\
& +\frac{1}{\Delta_{0}} \int_{a}^{x}\left[\lambda_{1}^{4}\left(\frac{t-a}{x-a}\right)^{\left|\lambda_{1}\right|}+\lambda_{2}^{4}\left(\frac{x-a}{t-a}\right)^{\lambda_{2}}+\lambda_{3}^{4}\left(\frac{x-a}{t-a}\right)^{\lambda_{3}}\right] \frac{f(t)}{t-a} \mathrm{~d} t .
\end{aligned}
$$

From equality (8) and (31) we find

$$
\begin{gathered}
C_{4}=\frac{-1}{\Delta_{0}} \lim _{x \rightarrow a}\left\{(x-a)^{-\lambda_{2}}\left[D_{x}^{a} \varphi(x)-\lambda_{3} \varphi(x)\right]\right\}=\frac{-1}{\Delta_{0}} \lim _{x \rightarrow a} T_{x}^{4} \varphi(x), \\
C_{5}=\frac{1}{\Delta_{0}} \lim _{x \rightarrow a}\left\{(x-a)^{-\lambda_{3}}\left[D_{x}^{a} \varphi(x)-\lambda_{2} \varphi(x)\right]\right\}=\frac{1}{\Delta_{0}} \lim _{x \rightarrow a} T_{x}^{5} \varphi(x) .
\end{gathered}
$$

From integral representation (10) it follows that if parameters $p_{j}(1 \leq j \leq 3)$ and function $f(x)$ in Equation (1) satisfy all condition of theorem 3 , then the solution of the type (10) has the property

$$
\left[(x-a)^{-\lambda_{3}} \varphi(x)\right]_{x=a}=C_{6} .
$$

From integral representation (14) it follows that

$$
\begin{gathered}
D_{x}^{a} \varphi(x)=(x-a)^{\lambda}\left[\lambda C_{1}+(1+\lambda \ln (x-a)) C_{2}+\left(2 \ln (x-a)+\lambda \ln ^{2}(x-a)\right) C_{3}\right]+D_{x}^{a} f(x) \\
+3 \lambda f(x)+\frac{\lambda}{2} \int_{a}^{x}\left[12 \lambda+8 \lambda \ln \left(\frac{x-a}{t-a}\right)+\lambda^{3} \ln ^{2}\left(\frac{x-a}{t-a}\right)\right]\left(\frac{x-a}{t-a}\right)^{\lambda} \frac{f(t)}{t-a} \mathrm{~d} t, \\
\left(D_{x}^{a}\right)^{2} \varphi(x)=(x-a)^{\lambda}\left[\lambda^{2} C_{1}+\left(2 \lambda+\lambda^{2} \ln (x-a)\right) C_{2}+\left(2+4 \lambda \ln (x-a)+\lambda^{2} \ln ^{2}(x-a)\right) C_{3}\right]+D_{x}^{a} f(x)+3 \lambda D_{x}^{a} f(x) \\
+\lambda^{2} f(x)+\frac{\lambda}{2} \int_{a}^{x}\left[8 \lambda+12 \lambda^{2}+\left(8 \lambda^{2}+2 \lambda^{3}\right) \ln \left(\frac{x-a}{t-a}\right)+\lambda^{4} \ln ^{2}\left(\frac{x-a}{t-a}\right)\right]\left(\frac{x-a}{t-a}\right)^{\lambda} \frac{f(t)}{t-a} \mathrm{~d} t .
\end{gathered}
$$

Using the formulas (14), (35) and (36), we easily see that, when fulfillment any condition of theorem 5, then solution of the type (14) has the following properties:

$$
\begin{aligned}
C_{1}= & \left\{\operatorname { l i m } _ { x \rightarrow a } ( x - a ) ^ { - \lambda } \left[\ln ^{2}(x-a)(\varphi(x))-2 \ln (x-a)(1+\ln (x-a))\left(D_{x}^{\alpha}\right)^{2}\right.\right. \\
& \left.\left.\cdot D_{x}^{\alpha}(\varphi(x))+\left(2+2 \lambda \ln (x-a)-\lambda^{2} \ln ^{2}(x-a)\right) \varphi(x)\right]\right\} \equiv \lim _{x \rightarrow a} T_{x}^{6}(\varphi(x)) \\
C_{2}= & -\lim _{x \rightarrow a}\left\{( x - a ) ^ { - \lambda } \left[2 \ln (x-a)\left(D_{x}^{\alpha}\right)^{2}(\varphi(x))-(2+2 \ln (x-a)\right.\right. \\
& \left.\left.\cdot(2 \lambda-1)+\ln ^{2}(x-a)\left(\lambda^{2}-\lambda\right) D_{x}^{\alpha}(\varphi(x))+\left(2 \lambda+2 \lambda^{2} \ln (x-a)\right) \varphi(x)\right]\right\} \equiv-\lim _{x \rightarrow a} T_{x}^{7}(\varphi(x)) \\
C_{3}= & \lim _{x \rightarrow a}\left\{(x-a)^{-\lambda}\left[\left(D_{x}^{\alpha}\right)^{2}(\varphi(x))-2 \lambda D_{x}^{\alpha}(\varphi(x))+\lambda^{2} \varphi(x)\right]\right\} \equiv \lim _{x \rightarrow a} T_{x}^{8}(\varphi(x)) .
\end{aligned}
$$

From integral representation (18) it follows that

$$
\begin{aligned}
& D_{x}^{\alpha} \varphi(x)=\lambda_{1}(x-a)^{\lambda_{1}} C_{1}+(x-a)^{A} . \\
\left\{\left[C_{2}(A \cos [B \ln (x-a)]-B \sin [B \ln (x-a)])+C_{3}(A \sin [B \ln (x-a)]+B \cos [B \ln (x-a)])\right]\right\}-\frac{\lambda_{1+D_{2}}^{3} f(x)+D_{x}^{\alpha} f(x)}{\Delta_{0}} & +\frac{1}{\Delta_{0}} \int_{a}^{x}\left\{\lambda_{1}^{4} B\left(\frac{x-a}{t-a}\right)^{\lambda_{1}}+\left(\frac{x-a}{t-a}\right)^{A}\left[\left(A D_{1}-D_{2} B\right) \sin \left[B \ln \left(\frac{x-a}{t-a}\right)\right]+\left(A D_{2}+D_{1} B\right) \cos \left[B \ln \left(\frac{x-a}{t-a}\right)\right]\right]\right\} \frac{f(t)}{t-a} \mathrm{~d} t,
\end{aligned}
$$




$$
\begin{aligned}
& \left(D_{x}^{a}\right)^{2} \varphi(x)=\lambda_{1}^{2}(x-a)^{\lambda_{1}} C_{1}+(x-a)^{A} \cdot\left\{\left[C_{2}\left(\left[A^{2}-B^{2}\right] \cos [B \ln (x-a)]-2 A B \sin [B \ln (x-a)]\right)\right.\right. \\
& \left.\left.+C_{3}\left(\left[A^{2}-B^{2}\right]+2 A B \sin [B \ln (x-a)]+B \cos [B \ln (x-a)]\right)\right]\right\} \\
& -\frac{\lambda_{1}^{4}+A D_{2}+B D_{1}}{\Delta_{0}} f(x)-\frac{\lambda_{1}^{2}+D_{2}}{\Delta_{0}} D_{x}^{\alpha} f(x)+\left(D_{x}^{a}\right)^{2} f(x)-\frac{1}{\Delta_{0}} \int_{a}^{x}\left\{\lambda_{1}^{5} B\left(\frac{x-a}{t-a}\right)^{\lambda_{1}}+\left(\frac{x-a}{t-a}\right)^{A}\right. \\
& \left.\left[\left(\left[A^{2}-B^{2}\right] D_{1}-2 A D_{2} B\right) \sin B \ln \left(\frac{x-a}{t-a}\right)+\left(\left[A^{2}-B^{2}\right] D_{2}+2 A D_{1} B\right) \cos B \ln \left(\frac{x-a}{t-a}\right)\right]\right\} \frac{f(t)}{t-a} \mathrm{~d} t .
\end{aligned}
$$

Using the formulas (18), (40) and (41), we easily see that, when fulfillment any condition of theorem 7, then solution of the type (18) has the following properties:

$$
\begin{aligned}
C_{1}= & \frac{1}{\Delta_{0}} \lim _{x \rightarrow a}\left\{(x-a)^{-\lambda_{1}}\left[B\left(D_{x}^{a}\right)^{2} \varphi(x)-2 A B D_{x}^{\alpha} \varphi(x)+B\left(A^{2}+B^{2}\right) \varphi(x)\right]\right\} \equiv \frac{1}{\Delta_{0}} \lim _{x \rightarrow a} T_{x}^{10}(\varphi(x)), \\
C_{2}= & \frac{1}{\Delta_{0}} \lim _{x \rightarrow a}\left\{( x - a ) ^ { - A } \left[\left(D_{x}^{a}\right)^{2} \varphi(x)\left[\left(\lambda_{1}-A\right) \sin [B \ln (x-a)]-B \cos B[\ln (x-a)]\right]\right.\right. \\
& +D_{x}^{\alpha} \varphi(x)\left[\left\{A^{2}-B^{2}-\lambda_{1}^{2}\right\} \sin [B \ln (x-a)]+2 A B \cos [B \ln (x-a)]\right]+\varphi(x) . \\
& {\left[\left\{\lambda_{1}^{2} A-\lambda_{1}\left(A^{2}-B^{2}\right)\right\} \sin [B \ln (x-a)]+\left\{\lambda_{1}^{2} B-2 A B\right\} \cos [B \ln (x-a)]\right] \equiv \frac{1}{\Delta_{0}} \lim _{x \rightarrow a} T_{x}^{11}(\varphi(x)), } \\
C_{3}= & \frac{1}{\Delta_{0}} \lim _{x \rightarrow a}\left\{( x - a ) ^ { - A } \left[\left(D_{x}^{a}\right)^{2} \varphi(x)\left[\left(A-\lambda_{1}\right) \cos [B \ln (x-a)]-B \sin [B \ln (x-a)]\right]\right.\right. \\
& -D_{x}^{\alpha} \varphi(x)\left[\left\{A^{2}-B^{2}-\lambda_{1}^{2}\right\} \cos [B \ln (x-a)]+2 A B \sin [B \ln (x-a)]\right]-\varphi(x) . \\
& {\left[\left\{\lambda_{1}^{2} A-\lambda_{1}\left(A^{2}-B^{2}\right)\right\} \cos [B \ln (x-a)]-\left\{\lambda_{1}^{2} B-2 A B\right\} \cos B[\ln (x-a)]\right] \equiv \frac{1}{\Delta_{0}} \lim _{x \rightarrow a} T_{x}^{12}(\varphi(x)) . }
\end{aligned}
$$

Differentiating the solution of the type (20), immediate verification, we can easily convince to correctness of the following equality:

$$
\begin{aligned}
D_{x}^{\alpha} \varphi(x)= & (x-a)^{A}\left[C_{2}(A \cos [B \ln (x-a)]-B \sin [B \ln (x-a)])\right. \\
& \left.+C_{3}(A \sin [B \ln (x-a)]+B \cos [B \ln (x-a)])\right]-\frac{\lambda_{1}^{3} B+D_{2}}{\Delta_{0}} f(x)+D_{x}^{\alpha} f(x) \\
& -\frac{1}{\Delta_{0}} \int_{a}^{x}\left\{\lambda_{1}^{4} B\left(\frac{t-a}{x-a}\right)^{\left|\lambda_{1}\right|}+\left(\frac{x-a}{t-a}\right)^{A}\left[\left(A D_{1}-B D_{2}\right) \sin \left[B \ln \left(\frac{x-a}{t-a}\right)\right]+\left(A D_{2}+B D_{1}\right) \cos \left[B \ln \left(\frac{x-a}{t-a}\right)\right]\right\} \frac{f(t)}{t-a} \mathrm{~d} t,\right.
\end{aligned}
$$

Using the formulas (20) and (45), we easily see that, when fulfillment any condition of theorem 8 , then solution of the type (20) has the following properties:

$$
\begin{aligned}
C_{2} & =\frac{1}{B} \lim _{x \rightarrow a}\left\{(x-a)^{-A}\left[A \sin [B \ln (x-a)]+B \cos [B \ln (x-a)] \varphi(x)-\sin [B \ln (x-a)] D_{x}^{\alpha} \varphi(x)\right]\right\} \\
& \equiv \frac{1}{B} \lim _{x \rightarrow a} T_{x}^{13}(\varphi(x)) . \\
C_{3} & =\frac{1}{B} \lim _{x \rightarrow a}\left\{(x-a)^{-A}\left[-\left[A \cos [B \ln (x-a)]+B \sin [B \ln (x-a)] \varphi(x)+\cos [B \ln (x-a)] D_{x}^{\alpha} \varphi(x)\right]\right]\right\} \\
& \equiv \frac{1}{B} \lim _{x \rightarrow a} T_{x}^{14}(\varphi(x)) .
\end{aligned}
$$


From integral representation (22) it follows that if parameters $p_{j}(1 \leq j \leq 3)$ and function $f(x)$ in equation (1) satisfy all condition of theorem 9 , then the solution of the type (22) has the property

$$
\left[(x-a)^{-\lambda_{1}} \varphi(x)\right]_{x=a}=C_{1} .
$$

\section{Boundary Value Problems}

When, the general solution constants, arbitrary constants higher mentioned properties of the solution the integral Equation (1) give possibility for integral Equation (1) put and investigate the following boundary value problems:

Problem $N_{1}$. Is required found the solution of the integral Equation (1) from class $C(\bar{\Gamma})$, when the roots the algebraic Equation (4) real, different and positive by boundary conditions

$$
\left\{\begin{array}{l}
{\left[T_{x}^{1} \varphi(x)\right]_{x=a}=A_{11}} \\
{\left[T_{x}^{2} \varphi(x)\right]_{x=a}=A_{12}} \\
{\left[T_{x}^{3} \varphi(x)\right]_{x=a}=A_{13},}
\end{array}\right.
$$

where $A_{11}, A_{12}, A_{13}$-are given constants.

Problem $N_{2}$. Is required found the solution of the integral Equation (1) from class $C(\bar{\Gamma})$, when the roots the algebraic Equation (4) real, different and also $\lambda_{1}<0$, $\lambda_{2}>0, \lambda_{3}>0$, by boundary conditions

$$
\left\{\begin{array}{l}
{\left[T_{x}^{4} \varphi(x)\right]_{x=a}=A_{21}} \\
{\left[T_{x}^{5} \varphi(x)\right]_{x=a}=A_{22},}
\end{array}\right.
$$

where $A_{21}, A_{22}$-are given constants.

Problem $N_{3}$. Is required found the solution of the integral Equation (1) from class $C(\bar{\Gamma})$, when the roots the algebraic Equation (4) real, different and also $\lambda_{1}<0$, $\lambda_{2}<0, \lambda_{3}>0$ by boundary conditions

$$
\left[(x-a)^{-\lambda_{3}} \varphi(x)\right]_{x=a}=A_{31},
$$

where $A_{31}$-are given constant.

Problem $N_{4}$. Is required found the solution of the integral Equation (1) from class $C(\bar{\Gamma})$, when the roots the algebraic Equation (4) real, equal and positive, that is $\lambda_{1}=\lambda_{2}=\lambda_{3}=\lambda>0$ by boundary conditions

$$
\left\{\begin{array}{l}
{\left[T_{x}^{6} \varphi(x)\right]_{x=a}=A_{41}} \\
{\left[T_{x}^{7} \varphi(x)\right]_{x=a}=A_{42}} \\
{\left[T_{x}^{8} \varphi(x)\right]_{x=a}=A_{43},}
\end{array}\right.
$$

where $A_{41}, A_{42}, A_{43}$-are given constants.

Problem $N_{5}$. Is required found the solution of the integral Equation (1) from class $\mathrm{C}(\bar{\Gamma})$, when the one roots of the algebraic Equation (4) real positive, two out of its complex-conjugate. Besides $A=$ Real $\lambda_{2}>0$, by boundary conditions

$$
\left\{\begin{array}{l}
{\left[T_{x}^{9} \varphi(x)\right]_{x=a}=A_{51}} \\
{\left[T_{x}^{10} \varphi(x)\right]_{x=a}=A_{52}} \\
{\left[T_{x}^{11} \varphi(x)\right]_{x=a}=A_{53},}
\end{array}\right.
$$

where $A_{51}, A_{52}, A_{53}$-are given constants.

Problem $N_{6}$. Is required found the solution of the integral Equation (1) from class $\mathrm{C}(\bar{\Gamma})$, when the one roots of the algebraic Equation (4) real positive, two out of its complex-conjugate. Besides $\lambda_{1}<0, A=$ Real $\lambda_{2}>0$, by boundary conditions

$$
\left\{\begin{array}{l}
{\left[T_{x}^{12} \varphi(x)\right]_{x=a}=A_{61}} \\
{\left[T_{x}^{13} \varphi(x)\right]_{x=a}=A_{62},}
\end{array}\right.
$$

where $A_{61}, A_{62}$-are given constants.

Problem $N_{7}$. Is required found the solution of the integral Equation (1) from class $\mathrm{C}(\bar{\Gamma})$, when the one roots of the algebraic Equation (4) real positive, two out of its complex-conjugate. Besides, $\lambda_{1}>0, A=\operatorname{Real} \lambda_{2}<0$, by boundary conditions

$$
\left[(x-a)^{-\lambda_{1}} \varphi(x)\right]_{x=a}=A_{71},
$$

where $A_{71}$-are given constant.

Solution problem $N_{1}$. Let fulfillment any condition of theorem 1. Then using the solution of the type (6) and its properties (28)-(30) and condition (49), we have

$$
C_{1}=\frac{\left(\lambda_{3}-\lambda_{2}\right)}{\Delta_{0}} A_{11}, C_{2}=\frac{\left(\lambda_{1}-\lambda_{3}\right)}{\Delta_{0}} A_{12}, C_{2}=\frac{\left(\lambda_{2}-\lambda_{3}\right)}{\Delta_{0}} A_{13}
$$

Substituting obtained valued $C_{1}, C_{2}$ and $C_{3}$ in formula (6), we find the solution of problem $N_{1}$ in form

$$
\begin{aligned}
& \varphi(x) \\
& =K_{1}^{-}\left[\frac{\left(\lambda_{3}-\lambda_{2}\right)}{\Delta_{0}} A_{11}, \frac{\left(\lambda_{1}-\lambda_{3}\right)}{\Delta_{0}} A_{12}, \frac{\left(\lambda_{2}-\lambda_{3}\right)}{\Delta_{0}} A_{13}, f(x)\right] .
\end{aligned}
$$

So, we proof.

Theorem 11. Let in integral Equation (1) parameters $p_{j}(1 \leq j \leq 3)$, function $f(x)$ satisfy any condition of theorem 1. Then Problem $N_{1}$ has a uniquesolution which is given by formula (56).

Solution problem $\boldsymbol{N}_{2}$. Let fulfillment any condition of theorem 2. Then using the solution of the type (8) and its properties (32), (33) and condition (50), we have:

$C_{4}=-\frac{1}{\Delta_{0}} A_{21}, C_{5}=\frac{1}{\Delta_{0}} A_{22}$. Substituting this valued $C_{4}$, $C_{5}$ in formula (8), we find the solution of problem $N_{2}$ in form 


$$
\varphi(x)=K_{2}^{-}\left[-\frac{1}{\Delta_{0}} A_{21}, \frac{1}{\Delta_{0}} A_{22}, f(x)\right] .
$$

So, we proof.

Theorem 12. Let in integral Equation (1) parameters $p_{j}(1 \leq j \leq 3)$, function $f(x)$ satisfy condition of theorem 2. Then problem $N_{2}$ has unique solution which is given by formula (57).

Solution problem $N_{3}$. Let fulfillment any condition of theorem 3. Then using the solution of the type (10) and its properties (32) and condition (51), we have: $C_{6}=A_{31}$. Substitute this valued $C_{6}$ in formula (10), we find thesolution of problem $N_{3}$ in form

$$
\varphi(x)=K_{3}^{-}\left[A_{31}, f(x)\right] .
$$

So, we proof.

Theorem 13. Let in integral Equation (1) parameters $p_{j}(1 \leq j \leq 3)$, function $f(x)$ satisfy condition of Theorem 3. Then problem $N_{3}$ has unique solution, which is given by formula (58).

Solution problem $N_{4}$. Let fulfillment any condition of theorem 5. Then using solution of the type (14) and its properties (37)-(39) and condition (52), we have: $C_{1}=$ $A_{41}, C_{2}=A_{42}, C_{3}=A_{43}$ Substituting this valued $C_{1}$, $C_{2}$ and $C_{3}$ in formula (14), we find thesolution of problem $N_{4}$ in form

$$
\varphi(x)=K_{5}^{-}\left[A_{41}, A_{42}, A_{43}, f(x)\right] .
$$

So, we proof.

Theorem 14. Let in integral Equation (1) parameters $p_{j}(1 \leq j \leq 3)$, function $f(x)$ satisfy condition of theorem 5. Then problem $N_{4}$ has unique solution, which is given by formula (59).

Solution problem $N_{5}$. Let fulfillment any condition of theorem 7. Then using solution of the type (18) and its properties (42)-(44), and condition (53) we have:

$C_{1}=\frac{1}{\Delta_{0}} A_{51}, \quad C_{2}=\frac{1}{\Delta_{0}} A_{52}, \quad C_{3}=\frac{1}{\Delta_{0}} A_{53}$. Substituting this valued $C_{1}, C_{2}$ and $C_{3}$ in formula (18) we findthe solution of problem $N_{5}$ in form

$$
\varphi(x)=K_{7}^{-}\left[A_{51}, A_{52}, A_{53}, f(x)\right] .
$$

So, we proof.

Theorem 15. Let in integral Equation (1) parameters $p_{j}(1 \leq j \leq 3)$, function $f(x)$ satisfy condition theorem

7. Then problem $N_{5}$ have unique solution, which is given by formula (60).

Solution problem $N_{6}$. Letfulfillment any condition of theorem 8. Then using solution of the type (20) and its properties (46), (47) and condition (54) we have:

$C_{2}=\frac{1}{B} A_{61}, C_{3}=\frac{1}{B} A_{62}$. Substituting this valued $C_{2}$ and
$C_{3}$ in formula (20) we find the solution of problem $N_{6}$ in form

$$
\varphi(x)=K_{8}^{-}\left[A_{61}, A_{62}, f(x)\right] .
$$

So, we proof.

Theorem 16. Let in integral Equation (1) parameters $p_{j}(1 \leq j \leq 3)$, function $f(x)$ satisfy condition theorem 8. Then problem $N_{6}$ have unique solution, which is given by formula (61).

Solution problem $N_{7}$. Let fulfillment any condition of theorem 9. Then using solution of the type (22) and its properties (48) and condition (55) we have: $C_{1}=A_{71}$. Substituting this value $C_{1}$ in formula (22) we find the solution of problem $N_{7}$ in form

$$
\varphi(x)=K_{9}^{-}\left[A_{71}, f(x)\right] .
$$

So, we proof.

Theorem 17. Let in integral Equation (1) parameters $p_{j}(1 \leq j \leq 3)$, function $f(x)$ satisfy condition theorem 9. Then problem $N_{7}$ have unique solution, which is given by formula (62).

\section{Presentation the Solution of the Integral Equation (1) in the Generalized Power Series}

Suppose that $f(x)$ has uniformly convergent power series expansion on $\Gamma$ :

$$
f(x)=\sum_{k=0}^{\infty}(x-a)^{k+\gamma} f_{k},
$$

where $\gamma=$ constant $>0$ and $f_{k}, k=0,1,2, \cdots$, are given constants. We attempt to find a solution of (1) in the form

$$
\varphi(x)=\sum_{k=0}^{\infty}(x-a)^{k+\gamma} \varphi_{k},
$$

where the coefficients, $\varphi_{k}(k=0,1,2, \cdots)$ are unknown.

Substituting power series representations of value $f(x)$ and $\varphi(x)$ into (1), equating the coefficients of the corresponding function, and for $\varphi_{k}$, we obtain

$$
\varphi_{k}=\frac{(k+\gamma)^{3}}{(k+\gamma)^{3}+p_{1}(k+\gamma)^{2}+p_{2}(k+\gamma)+2 p_{3}} f_{k},
$$

$$
k=0,1,2,3, \cdots \text {. }
$$

If $(k+\gamma)^{3}+p_{1}(k+\gamma)^{2}+p_{2}(k+\gamma)+2 p_{3} \neq 0$ for in all $k=0,1,2, \cdots$, putting the found coefficients back into (64), we arrive at the particular solution of (1).

$$
\begin{aligned}
& \varphi(x) \\
& =\sum_{k=0}^{\infty}(x-a)^{k+\gamma} \frac{(k+\gamma)^{3}}{(k+\gamma)^{3}+p_{1}(k+\gamma)^{2}+p_{2}(k+\gamma)+2 p_{3}} f_{k} .
\end{aligned}
$$

If, for some values $k=k_{1}, k=k_{2}$ and $k=k_{3}$, con- 
stants $\gamma, p_{j}(1 \leq j \leq 3)$ satisfy

$$
(k+\gamma)^{3}+p_{1}(k+\gamma)^{2}+p_{2}(k+\gamma)+2 p_{3}=0,
$$

then the solution to integral Equation (1) can be represented in the form (64) it is necessary and sufficiently that $f_{k_{j}}=0, j=1,2,3$, that is, it is necessary and sufficiently that function $f(x)$ in point $x=a$ satisfies the following three solvability condition

$$
\left[\left[(x-a)^{-\gamma} f(x)\right]^{\left(k_{j}\right)}\right]_{x=a}=0, j=1,2,3 .
$$

In this case the solution of the integral Equation (1) in the class of function can be represented in form (64) is given by formula

$$
\begin{aligned}
\varphi(x)= & \sum_{k=0}^{k_{1}-1}(x-a)^{k+\gamma} \frac{(k+\gamma)^{3}}{(k+\gamma)^{3}+p_{1}(k+\gamma)^{2}+p_{2}(k+\gamma)+2 p_{3}} f_{k} \\
& +\sum_{k=k_{1}+1}^{k_{3}-1}(x-a)^{k+\gamma} \frac{(k+\gamma)^{3}}{(k+\gamma)^{3}+p_{1}(k+\gamma)^{2}+p_{2}(k+\gamma)+2 p_{3}} f_{k} \\
& +\sum_{k=k_{3}+1}^{\infty}(x-a)^{k+\gamma} \frac{(k+\gamma)^{3}}{(k+\gamma)^{3}+p_{1}(k+\gamma)^{2}+p_{2}(k+\gamma)+2 p_{3}} f_{k} \\
& +\varphi_{k_{1}}(x-a)^{k_{1}}+\varphi_{k_{2}}(x-a)^{k_{2}}+\varphi_{k_{3}}(x-a)^{k_{3}}
\end{aligned}
$$

where $\varphi_{k_{1}}, \varphi_{k_{2}}, \varphi_{k_{3}}$ arbitrary constants.

Immediately testing it we see that, if converges radius of the series (63) is defined by formula $R=\frac{1}{l}$,

$l=\lim _{n \rightarrow \infty} \frac{\left|f_{n+1}\right|}{\left|f_{n}\right|}$, then converges radius of the series (66),

(68) are also defined by this formula. So, we prove the next result.

Theorem 18. Let in integral Equation (1), function $f(x)$ represent in formuniformly-converges generalized power series type (63) and

$$
(k+\gamma)^{3}+p_{1}(k+\gamma)^{2}+p_{2}(k+\gamma)+2 p_{3} \neq 0,
$$

for $k=0,1,2, \cdots$. Then integral Equation (1) in class of function $\varphi(x)$ represented in form (64) has a uniquesolution, which is given by formula (66). For values $k=k_{j}, \quad j=1,2,3$,

$$
(k+\gamma)^{3}+p_{1}(k+\gamma)^{2}+p_{2}(k+\gamma)+2 p_{3}=0
$$

the existence of the solution of Equation (1) can be represented in form (64) it is necessary and sufficiently fulfillment three solvability condition type (67). In this case integral Equation (1) in class of function represented in form (63) is always solvability and its general solution contain tree arbitrary constants and is given by formula (68).

\section{General Case}

In general case to integral Equation $(I)$ corresponding the following algebraic equation

$$
\begin{aligned}
& \lambda^{n}+p_{1} \lambda^{n-1}+p_{2} \lambda^{n-2}+2 ! p_{3} \lambda^{n-3} \\
& +3 ! p_{4} \lambda^{n-4}+\ldots+(n-1) ! p_{n}=0 .
\end{aligned}
$$

Some results obtained in the general case to. Example in the case, when the roots of the Equation (II) real, different and positive have the following confirmation.

Theorem 19. Let in integral Equation (I) parmeters $p_{j}(1 \leq j \leq n)$ such that, the roots of the algebraic Equation (II) real, different and positive, function $f(x) \in$ $C(\bar{\Gamma}), f(a)=0$ with asymptotic behavior

$$
\begin{aligned}
& f(x)=o\left[(x-a)^{\delta_{1}}\right], \delta_{1}>\lambda, \\
& \lambda=\max \left(\lambda_{1}, \lambda_{2}, \cdots, \lambda_{n}\right) \text { at } x \rightarrow a,
\end{aligned}
$$

Then integral Equation $(I)$ in class of function $\varphi(x) \in$ $C(\bar{\Gamma})$ vanishing in point $x=a$ is always solvability and its solution is given by formula

$$
\begin{aligned}
\varphi(x)= & \sum_{k=1}^{n}(x-a)^{\lambda_{k}} C_{k} \\
& +f(x) \frac{1}{\Delta_{0}} \int_{a}^{x}\left[\sum_{k=1}^{n} \lambda_{k}^{3}\left(\frac{x-a}{t-a}\right)^{\lambda_{k}}\right] \frac{f(t)}{t-a} \mathrm{~d} t \\
\equiv & K_{1}^{-}\left[C_{1}, C_{2}, C_{3}, \cdots, C_{n}, f(x)\right],
\end{aligned}
$$

where $C_{k}(1 \leq k \leq n)$-arbitrary constants,

$$
\Delta_{0}=\left|\begin{array}{c}
1,1, \cdots, 1 \\
\lambda_{1}, \lambda_{2}, \cdots \lambda_{n} \\
\lambda_{1}^{2}, \lambda_{2}^{2}, \cdots, \lambda_{n}^{2} \\
\cdots \\
\cdots \\
\lambda_{1}^{n-1}, \lambda_{2}^{n-1}, \cdots, \lambda_{n}^{n-1}
\end{array}\right| .
$$

\section{Conclusions}

So, in this article we consider new class Volterra type integral equation, which no submitting exists Fredholm theory (Theory Volterra type integral equation in class 
$\left.C(\bar{\Gamma}), L_{2}(\bar{\Gamma})\right)$, that is for this type integral equation, homogeneous integral equation may have non-zero solution. In particular in certain cases (Example, when all roots of the characteristic Equation (4) or (II) real, different negative or real, equal and negative) the theory this type integral equation coincides to the theory Fredholm integral equation.

By means methods (example [5]) in the theory one dimensional singular integral equation, problem finding the solution general equation

$$
\begin{aligned}
& \varphi(x)+\int_{a}^{x}\left[\sum_{m=1}^{n} K_{m}(x, t) \ln ^{m-1}\left(\frac{x-a}{t-a}\right)\right] \frac{\varphi(t)}{t-a} \mathrm{~d} t \\
& =f(x),
\end{aligned}
$$

reduces to finding solution Volterra type integral equation with weak singularity. On this basis, in depend from roots of the algebraic equation

$$
\lambda^{n}+K_{1}(a, a) \lambda^{n-1}+K_{2}(a, a) \lambda^{n-2}+2 ! K_{3}(a, a) \lambda^{n-3}+3 ! K_{4}(a, a) \lambda^{n-4}+\cdots+(n-1) ! K_{n}(a, a)=0,
$$

$K_{m}(a, a) \neq 0(1 \leq m \leq n)$, select cases, when general solution equation contains $n, n-1, n-2, \cdots, 1$ arbitrary constants, and cases when Equation (IV) has unique solu- tion.

In this case, integral Equation (IV), we represented to following form

$$
\varphi(x)+\int_{a}^{x}\left[\sum_{m=1}^{n} K_{m}(a, a) \ln ^{m-1}\left(\frac{x-a}{t-a}\right)\right] \frac{\varphi(t)}{t-a} \mathrm{~d} t=F(x),
$$

where

$$
F(x)=f(x)-\int_{a}^{x}\left[\sum_{m=1}^{n}\left(K_{m}(x, t)-K_{m}(a, a)\right) \ln ^{m-1}\left(\frac{x-a}{t-a}\right)\right] \frac{\varphi(t)}{t-a} \mathrm{~d} t .
$$

According to the mentioned above, writing the solution integral Equation $(V I)$ in depend to the roots of the characteristic Equation $(V)$ or $(I I)$, after substituting for $F(x)$ from formula $(V I I)$ we arrive at the solution of the new type integral equation. At specific condition to functions $K_{m}(x, t)-K_{m}(a, a)$ and $f(x)$ this integral equation will be Volterra type integral equation with weak singularity in point $=a$. In this basis the problem investigation integral Equation (IV), reduce to problem investigation Volterra type integral equation with weak singularity in point $x=a$.

\section{REFERENCES}

[1] N. Rajabov, "About One Three-Dimensional Volterra Type Integral Equation with Singular Boundary Surfaces in Kernels," Russian of Sc. Dokl, Vol. 409, No. 6, 2006, pp. 749-753.

[2] N. Rajabov, "On a Volterra Integral Equation," Doclady Mathematics, Vol. 65, No. 2, 2002, pp. 217-220.

[3] N. Rajabov, "System of Linear Integral Equations of Volterra Type with Singular and Super-singular Kernels," Ill-Posed and Non-Classical Problems of Mathematical Physics and Analysis. Proceedings of the International
Conference, Samarkand, Uzbekistan, Kluwer, Utrecht, Boston, 11-15 September 2000, pp. 103-124.

[4] N. Rajabov, "About One Class of Volterra Type Linear Integral Equations with an Interior Fixed Singular or Super-singular Point," Topics in Analysisand its Applications, NATO Science Series, II, Mathematics, Physics and Chemistry, Vol. 147, Kluwer Academic Publishers, 2004, pp. 317-326.

[5] N. Rajabov, "Volterra Type Integral Equation with Boundary and Interior Fixed Singularity and Super-Singularity Kernels and Their Application," LAPLAMBERT Academic Publishing, 2011, 282 p.

[6] N. Rajabov, "About New Class of Volterra Type Integral Equations with Boundary Singularity in Kernels," In $\mathrm{Ad}$ vances in Applied Mathematics and Approximation Theory: Contributions from AMAT2012, Springer, 2012, pp. 341-360.

[7] N. Rajabov, "To Theory One Class Modeling Linear Volterra Type Integral Equation with Boundary Singular Kernels," Theses of Reports of the 4th International Conference "Function Spaces. Differential Operators. General Topology. Problems of Mathematical Education", PFUR Publishers, Moscow, 2013, pp. 221-222. 